Article

\title{
Probability Functions on Posets
}

\author{
Jae Hee Kim $^{1}$, Hee Sik Kim ${ }^{1, * \mathbb{D}}$ and Joseph Neggers ${ }^{2}$ \\ 1 Research Institute for Natural Sciences, Department of Mathematics, Hanyang University, \\ Seoul 04763, Korea \\ 2 Department of Mathematics, University of Alabama, Tuscaloosa, AL 35487-0350, USA \\ * Correspondence: heekim@hanyang.ac.kr; Tel.: +82-2-2220-0897
}

Received: 11 July 2019; Accepted: 21 August 2019; Published: 25 August 2019

\begin{abstract}
In this paper, we define the notion of a probability function on a poset which is similar to the probability function discussed on $d$-algebras, and obtain three probability functions on posets. Moreover, we define a probability realizer of a poset, and we provide some examples to describe its role for the standard probability function. We apply the notion of a probability function to the ordered plane and obtain three probability functions on it.
\end{abstract}

Keywords: probability function; poset; $d$-algebra; probability realizer; ordered plane

\section{Introduction}

There has been some research between posets and algebraic structures. Neggers [1] proved that there is a natural isomorphism between the category of pogroupoids and the category of posets. Neggers and Kim [2] showed that a poset $(X, \leq)$ is $\left(C_{2}+\underline{1}\right)$-free if and only if its associated pogroupoid $(X, \cdot)$ is modular*. Neggers and Kim [3] introduced the notion of $d$-algebras. It is a kind of generalization of $B C K$-algebras to which they discussed some relations between $d$-algebras and $B C K$-algebras, as well as some relations between $d$-algebras and oriented digraphs. Cha et al. [4] introduced the notions of a trend and probability functions on $d$-algebras. They obtained an equivalent condition defining a trend $\pi_{0}$ with condition $(j)$ on a standard $B C K$-algebra. Loof et al. [5] discussed mutual rank probabilities in partially ordered sets. Baets et al. [6] characterized the transitivity of the mutual rank probability relation of a poset, and Lerche et al. [7] evaluated ranking probabilities for partial orders based on random linear extensions.

In this paper, we define a probability function on a poset. The idea of a probability function on a poset came from [4], and we obtained some probability functions on a poset. We defined a probability realizer on a poset, and found some examples for probability realizers of posets for the standard probability function $\pi_{0}$. Moreover, we applied the notion of a probability function to the ordered plane (order geometry), and found three probability functions acting on it. Some comments have been suggested for further research.

\section{Preliminaries}

Some definitions and terminologies will be recalled for partially ordered sets which are necessary for reading this paper.

An ordered pair $(X, \leq)$ is called a partially ordered set if $\leq$ is a partial order, i.e., reflexive, anti-symmetric, and transitive, on the set $X$. A poset $(X, \leq)$ is said to be a chain if every two distinct elements of $X$ are comparable, and we denote it by $C_{n}$ when the cardinality of $X$ is equal to $n$. A poset $(X, \leq)$ is said to be an anti-chain if every two distinct elements of $X$ are incomparable, and we denote it by $\underline{n}$ when the cardinality of $X$ is equal to $n$. Given two posets $X$ and $Y$, a poset $Z$ is said to be an ordinal sum of $X$ and $Y$ if $z_{1} \leq z_{2}$ in $Z$, then either $z_{1} \in X$ and $z_{2} \in Y$ or $z_{1} \leq z_{2}$ in $X$, or $z_{1} \leq z_{2}$ in $Y$. 
A graph $Z$ can be realized by placing the Hasse diagram of $Y$ above the Hasse diagram of $X$, and by drawing line segments from the maximal elements of $X$ to all the minimal elements of $Y$. We denote it by $Z=X \oplus Y$. A chain $\left(X, \leq^{*}\right)$ is said to be a linear extension of a poset $(X, \leq)$ if $x \leq y$ implies $x \leq^{*} y$. A family of linear extensions $R=\left\{L_{1}, \cdots, L_{k}\right\}$ of a poset $(X, \leq)$ is said to be a realizer of $(X, \leq)$ if $(X, \leq)$ can be realized as the intersection of $R$, but not as the intersection of fewer than $k$ linear extensions. For details we refer to [8].

A non-empty set $X$ with a constant 0 and a binary operation " $*$ " is said to be a $d$-algebra [3] if it satisfies: (i) $x * x=0$, (ii) $0 * x=0$, and (iii) $x * y=0$ and $y * x=0$ imply $x=y$ for all $x, y \in X$.

A mapping $\pi: X \times X \rightarrow[0,1]$ is said to be a trend [4] on a $d$-algebra $(X, *, 0)$ if it satisfies: for any $x, y \in X$,

(a) $x * y=0$ implies $\pi(x, y)=1$,

(b) $\quad x * y \neq 0$ implies $\pi(x, y)+\pi(y, x)=1$.

A trend $\pi: X \times X \rightarrow[0,1]$ is said to be a probability function [4] on a $d$-algebra $(X, *, 0)$ if it satisfies: for any $x, y, z \in X$,

(c) $\quad y * z=0$ implies $\pi(x, y) \leq \pi(x, z)$.

It is of course possible to consider other conditions to build (different) notions of trends and probability functions and to compare the resulting classes with those obtained here. In fact, we will actually do so below. As an example of the situation above, let $X:=[0, \infty)$ and let $x * y:=0$ if and only if $x \leq y$, and $x * y:=1$ otherwise. Thus (a) holds if $\pi(x, y):=x * y$. If $y \leq z$, then $x \leq y$ implies $x \leq z$ while $y>z$ means $\pi(y, z)=y * z=1$, so that (c) does not apply in that case. Condition (b) holds since $x * y \neq 0$ implies $x * y=1$, and $x>y$ yields $\pi(y, x)=1$ since $y<x$ in that case. The groupoid $(X, *, 0)$ is certainly a $d$-algebra.

\section{Probability Functions on Posets}

In this section we discuss probability functions on posets. We define the notion of a probability function on a poset which is a little bit different from the probability function discussed in $d$-algebras. Given a poset $P$, a map $\pi: P \times P \rightarrow[0,1]$ is called a probability function on $P$ if:

(I) $\quad \pi(x, x)=1$,

(II) $\quad x \leq y, x \neq y$ implies $\pi(x, y)=1$,

(III) $\quad x \neq y$ implies $\pi(x, y)+\pi(y, x)=1$,

(IV) $\quad y<z$ implies $\pi(x, y) \leq \pi(x, z)$,

for any $x, y, z \in X$.

Theorem 1. If $\pi$ is a probability function on a poset $(P, \leq)$, then:

$(\mathrm{IV})^{\prime} y<z$ implies $\pi(z, x) \leq \pi(y, x)$,

for any $x, y, z \in P$.

Proof. Let $y<z$ and let $x \in P$. Case (i): $x=z$. By (I), we have $\pi(z, x)=\pi(x, x)=1$ and $\pi(y, x)=\pi(y, z)=1$. Hence $\pi(z, x) \leq \pi(y, x)$. Case (ii): $z \neq x$. By (III), we have $\pi(z, x)+\pi(x, z)=1$. We have 3 subcases:



(ii-1) (ii-2) (ii-1) 
Subcase (ii-1): $z<x$. By (II), we have $\pi(z, x)=1$. Now, $y<z, z<x$ implies $y<x$ and hence $\pi(y, x)=1$. Hence $\pi(z, x) \leq \pi(y, x)$. Subcase (ii-2): $x<z$. By (III), $1=\pi(x, z)+\pi(z, x)=1+\pi(z, x)$, since $x \neq z$. It follows that $\pi(z, x)=0$. Hence $\pi(z, x)=0 \leq \pi(y, x)$. Subcase (ii-3): $z \| x$, i.e., $z$ and $x$

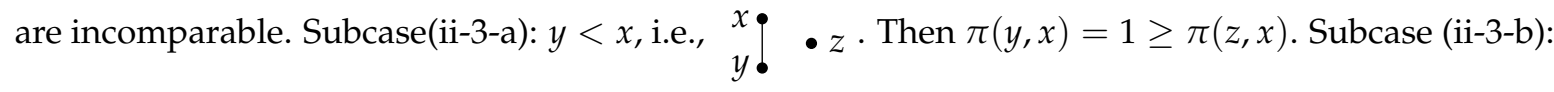
$x<y$. Since $y<z$, we have $: \begin{aligned} & z \\ & y\end{aligned}$, i.e., $x<z$. This is a contradiction, since $z \| x$. Subcase(ii-3-c): $x \| y$. Since $y<z$, by (IV), we have $\pi(x, y) \leq \pi(x, z)$. Since $z\|x, x\| y$, we have $z \neq x, x \neq y$, and $y<z$, whence it follows that $\begin{gathered}z \bullet \\ y \bullet\end{gathered}$. Hence $\pi(y, x)=1-\pi(x, y) \geq 1-\pi(x, z)=\pi(z, x)$. Thus $(\mathrm{IV})^{\prime}$ holds.

Remark 1 (Another proof of Theorem 1). This proof was suggested by a reviewer. It was proved axiomatically, not using the Hasse diagram of a poset. Let $y<z$ and let $x \in P$. We consider 3 cases: (i) Assume that $x \neq y$ and $x \neq z$. Then $\pi(x, y) \leq \pi(x, z)$. It follows that $\pi(y, x)=1-\pi(x, y) \geq 1-\pi(x, z)=\pi(z, x)$ by using the conditions (III) and (IV). (ii) Assume $x=y$. Then $\pi(y, x)=\pi(x, x)=1 \geq \pi(z, x)$. (iii) Assume $x=z$. Since $y<z=x$, by (II) we obtain $\pi(y, x)=1 \geq \pi(z, x)$, proving the theorem.

Notice that if $\alpha, \beta \in[0,1]$ and $\alpha+\beta=1$, then if $\pi_{1}$ and $\pi_{2}$ are probability functions on a poset $P$, it follows that $\alpha \pi_{1}+\beta \pi_{2}$ is also a probability function on $P$.

Let $(P, \leq)$ be a poset. Define a map $\pi_{0}: P^{2} \rightarrow[0,1]$ by:

$$
\pi_{0}(x, y):= \begin{cases}\frac{1}{2} & \text { if } x \| y, x \neq y \\ 0 & \text { if } y<x \\ 1 & \text { if } x \leq y\end{cases}
$$

for any $x, y \in P$. We shall prove such a function $\pi_{0}$ is a probability function on a poset $P$.

Proposition 1. The mapping $\pi_{0}$ is a probability function on $P$.

Proof. The conditions (I), (II), and (III) hold trivially. Assume that the condition (IV) does not hold. Then there exist $y, z \in P$ such that $y<z$ and $\pi_{0}(x, y)>\pi_{0}(x, z)$ for some $x \in P$. Case (i): $\pi_{0}(x, y)=1, \pi_{0}(x, z)=\frac{1}{2}$. Then $x \leq y, x \| z, x \neq z$. Since $y<z$, we have $x \leq z$, a contradiction. Case (ii): $\pi_{0}(x, y)=1, \pi_{0}(x, z)=0$. We have $x \leq y, z<x$, which implies $z \leq y$, which is a contradiction. Case (iii): $\pi_{0}(x, y)=\frac{1}{2}, \pi_{0}(x, z)=0$. Then $x \| y, x \neq y, z<x$. Since $y<z$, we have $x \| y, y<x$, which is a contradiction.

Remark 2. The reviewer suggested that the proof of Proposition 1 can be proved axiomatically. We omit its proof.

The mapping $\pi_{0}$ discussed in Proposition 1 is called a standard probability function.

Let $(P, \leq)$ be a finite poset and let $L(P)$ be the collection of all linear extensions of $P$. Given $x, y \in P$, we denote $L(P ; x \leq y)$ the collection of all linear extensions satisfying the condition $x \leq y$.

Theorem 2. Let $(P, \leq)$ be a finite poset. If we define a map $\pi_{L}: P^{2} \rightarrow[0,1]$ by:

$$
\pi_{L}(x, y):=\frac{|L(P ; x \leq y)|}{|L(P)|}
$$

for all $(x, y) \in P^{2}$, then $\pi_{L}$ is a probability function on $P$. 
Proof. (I) Given $x \in P$, we have $\pi_{L}(x, x)=\frac{|L(P ; x \leq x)|}{|L(P)|}=\frac{|L(P)|}{|L(P)|}=1$. (II) Let $x \leq y, x \neq y$ in $(P, \leq)$. Then $x<y$ in $L_{i}$ for any linear extension $L_{i}$ of $(P, \leq)$. It follows that $L(P ; x<y)=L(P)$, and hence $\pi_{L}(x, y)=\frac{|L(P ; x \leq y)|}{|L(P)|}=1$. (III) Let $x \neq y$ in $(P, \leq)$. Then, for any linear extension $L_{i}$ of $(P, \leq)$, either $x<y$ or $y<x$. This means that there exist $|L(P ; x<y)|$-number of linear extensions of $(P, \leq)$ and $|L(P ; y<x)|$-number of linear extensions of $(P, \leq)$. It follows that:

$$
\begin{aligned}
\pi_{L}(x, y)+\pi_{L}(y, x) & =\frac{|L(P ; x<y)|+|L(P ; y<x)|}{|L(P)|} \\
& =\frac{|L(P ; x<y)|+(|L(P)|-|L(P ; x<y)|)}{|L(P)|} \\
& =1 .
\end{aligned}
$$

(IV) Let $y<z$ in $(P, \leq)$ and let $x \in P$. If $L_{i}$ is a linear extension of $P$ with $x<y$, then $L_{i}$ is also a linear extension of $P$ with $x<z$, since $y<z$. It follows that $|L(P ; x<y)| \leq|L(P ; x<z)|$. Hence:

$$
\begin{aligned}
\pi_{L}(x, y) & =\frac{|L(P ; x<y)|}{|L(P)|} \\
& \leq \frac{|L(P ; x<z)|}{|L(P)|} \\
& =\pi_{L}(x, z) .
\end{aligned}
$$

This proves the theorem.

Example 1. Let $P:=\{x, y, z\}$ be a poset with the diagram:

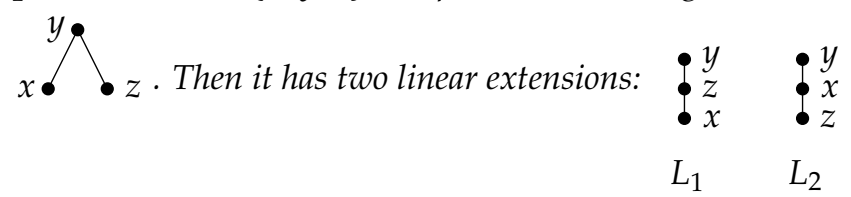

It follows that $L(P)=\left\{L_{1}, L_{2}\right\}$ and $L(P ; x \leq z)=\left\{L_{1}\right\}$. Hence $\pi_{L}(x \leq z)=\frac{1}{2}$.

Corollary 1. If $(P, \leq)$ is an anti-chain, then $\pi_{L}=\pi_{0}$.

Proof. If $(P, \leq)$ is an anti-chain, then $|L(P, x \leq y)|=\frac{1}{2}|L(P)|$ and hence $\pi_{L}(P ; x \leq y)=\frac{1}{2}$. This shows that $\pi_{L}=\pi_{0}$.

Let $(P, \leq)$ be a poset and let $\bigwedge$ be a linear extension of $(P, \leq)$. Define a map $\pi_{\wedge}$ on $P^{2}$ by:

$$
\pi_{\wedge}(x, y):= \begin{cases}1 & \text { if } x \leq y \text { in } \wedge \\ 0 & \text { otherwise. }\end{cases}
$$

Proposition 2. Let $(P, \leq)$ be a poset and let $\wedge$ be a linear extension of $(P, \leq)$. Then $\pi_{\wedge}$ is a probability function on $P$.

Proof. We show the condition (IV). Let $y<z$. For any $x \in P$ with $x<y$, we have $x<z$. Hence $\pi_{\wedge}(x, y)=1$ implies $\pi_{\wedge}(x, z)=1$, which implies that $\pi_{\wedge}(x, y) \leq \pi_{\wedge}(x, z)$.

Theorem 3. Let $(P, \leq)$ be a finite poset and let $n:=|L(P)|$, say $L(P)=\left\{\wedge_{1}, \wedge_{2}, \cdots, \wedge_{n}\right\}$. Then $\pi_{L}=\frac{1}{n} \sum_{i=1}^{n} \pi_{\bigwedge_{i}}$ 
Proof. Given $x, y \in P$, we have 3 cases. Case $1: x \leq y$ in $(P, \leq)$. Then $|L(P ; x \leq y)|=|L(P)|$ and hence $\pi_{L}(x, y)=1$. Since $x \leq y$ in $(P, \leq)$ implies $x \leq y$ in $\bigwedge_{i}$ for any $i \in\{1,2, \cdots, n\}$, we have $\frac{1}{n} \sum_{i=1}^{n} \pi_{\Lambda_{i}}(x, y)=\frac{1}{n} \sum_{i=1}^{n} 1=1$. Case $2: y \leq x$ in $(P, \leq)$. If $y<x$ in $(P, \leq)$, then $y<x$ in $\bigwedge_{i}$ for any $i \in\{1,2, \cdots, n\}$. It follows that $\pi_{L}(x, y)=0$ and $\pi_{\bigwedge_{i}}(x, y)=0$ for any $i \in\{1,2, \cdots, n\}$. This shows that $\frac{1}{n} \sum_{i=1}^{n} \pi_{\Lambda_{i}}(x, y)=0=\pi_{L}(x, y)$. Case 3: $x \| y$ in $(P, \leq)$. Let $\left\{i_{1}, \cdots, i_{k}\right\} \subseteq\{1,2, \cdots, n\}$ such that $x \leq y$ in $\bigwedge_{i_{j}}$ where $j=1,2, \cdots, k$. It follows that $|L(P ; x \leq y)|=k$ and hence $\pi_{L}(x, y)=\frac{|L(P ; x \leq y)|}{|L(P)|}=\frac{k}{n}$. Since $\pi_{\bigwedge_{i}}(x, y)=0$ for any $i \in\{1,2, \cdots\} \backslash\left\{i_{1}, \cdots, i_{k}\right\}$, we obtain $\frac{1}{n} \sum_{i=1}^{n} \pi_{\bigwedge_{i}}(x, y)=\frac{1}{n} \sum_{j=1}^{k} \pi_{\bigwedge_{j}}(x, y)=\frac{k}{n}$, proving the theorem.

\section{Some Examples for Probability Realizers for $\pi_{0}$}

Let $(P, \leq)$ be a finite poset and let $B:=\left\{\bigwedge_{1}, \cdots, \wedge_{n}\right\}$ be a set of all linear extensions of $(P, \leq)$. A set $\left\{\pi_{\bigwedge_{i_{1}}}, \cdots, \pi_{\bigwedge_{i_{k}}} \mid \bigwedge_{i} \in B\right\}$ is said to be a probability realizer of $(P, \leq)$ for probability function $\pi$ if $\pi=\alpha_{1} \pi_{\bigwedge_{i_{1}}}+\alpha_{1} \pi_{\bigwedge_{i_{2}}}+\cdots+\alpha_{1} \pi_{\bigwedge_{i_{k}}}$ for some $\alpha_{1}, \cdots, \alpha_{k} \in[0,1]$.

Example 2. Let $\mathrm{N}:=\{1,2,3,4\}$ be a letter $\mathrm{N}$-poset:

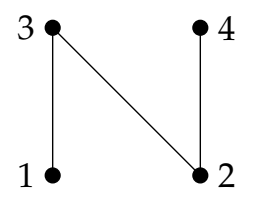

Then we have five linear extensions as follows:

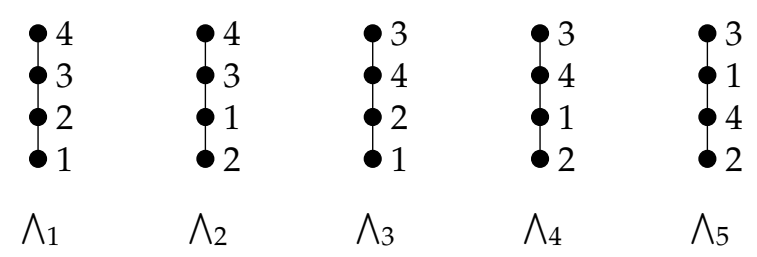

Consider $1 \| 4$ in $(\mathrm{N}, \leq)$. Assume $\pi_{0}=\alpha_{1} \pi_{\Lambda_{1}}+\cdots+\alpha_{5} \pi_{\Lambda_{5}}$. Then $\frac{1}{2}=\pi_{0}(1,2)=\alpha_{1} \pi_{\Lambda_{1}}(1,2)+\alpha_{2} \pi_{\Lambda_{2}}(1,2)+\alpha_{3} \pi_{\Lambda_{3}}(1,2)+\alpha_{4} \pi_{\Lambda_{4}}(1,2)+\alpha_{5} \pi_{\Lambda_{5}}(1,2)=\alpha_{1}+\alpha_{3}$. Similarly, we have $\alpha_{5}=\frac{1}{2}, \alpha_{1}+\alpha_{2}=\frac{1}{2}, \alpha_{1}+\alpha_{2}+\alpha_{3}+\alpha_{4}=\frac{1}{2}$. From this we obtain $\alpha_{1}=\alpha_{5}=\frac{1}{2}$ and $\alpha_{2}=\alpha_{3}=\alpha_{4}=0$. Hence $\pi_{0}=\frac{1}{2} \pi_{\Lambda_{1}}+\frac{1}{2} \pi_{\Lambda_{5}}$, i.e., $\left\{\pi_{\Lambda_{1}}, \pi_{\Lambda_{5}}\right\}$ is a probability realizer of letter $\mathrm{N}$-poset for the standard probability function $\pi_{0}$.

Example 3. Consider an anti-chain $\underline{3}:=\{1,2,3\}$. It has the following six linear extensions:

$\begin{array}{llllll}\bullet 3 & \bullet 2 & \bullet 1 & \bullet 1 & \bullet 2 & \bullet 2 \\ \bullet 2 & \bullet 1 & \bullet^{3} & \bullet 2 & \bullet 3 & \bullet^{3} \\ \bullet 1 & \bullet 3 & \bullet 2 & \bullet 3 & \bullet 1 & \bullet 1 \\ \wedge_{1} & \wedge_{2} & \wedge_{3} & \wedge_{4} & \wedge_{5} & \Lambda_{6}\end{array}$

Assume $\pi_{0}=\sum_{i=1}^{6} \alpha_{i} \pi_{\bigwedge_{i}}$ for some $\alpha_{i} \in[0,1]$. Then we have the following equations:

$$
\begin{aligned}
& \frac{1}{2}=\pi_{0}(1,2)=\alpha_{1}+\alpha_{2}+\alpha_{5}, \\
& \frac{1}{2}=\pi_{0}(1,3)=\alpha_{1}+\alpha_{5}+\alpha_{6}, \\
& \frac{1}{2}=\pi_{0}(2,3)=\alpha_{1}+\alpha_{3}+\alpha_{6}, \\
& \frac{1}{2}=\pi_{0}(2,1)=\alpha_{3}+\alpha_{4}+\alpha_{6} \\
& \frac{1}{2}=\pi_{0}(3,1)=\alpha_{2}+\alpha_{3}+\alpha_{4} \\
& \frac{1}{2}=\pi_{0}(3,2)=\alpha_{2}+\alpha_{4}+\alpha_{5} .
\end{aligned}
$$


From these equations, we obtain $\frac{1}{2}=\alpha_{1}+\alpha_{2}+\alpha_{5}=\alpha_{1}+\alpha_{2}+\alpha_{3}$. If we take $\alpha_{1}=\frac{1}{2}, \alpha_{2}=\alpha_{3}=0$, then $\pi_{0}=\frac{1}{2}\left(\pi_{\Lambda_{1}}+\pi_{\Lambda_{4}}\right)$. If we take $\alpha_{2}=\frac{1}{2}, \alpha_{1}=\alpha_{3}=0$, then $\pi_{0}=\frac{1}{2}\left(\pi_{\Lambda_{2}}+\pi_{\Lambda_{6}}\right)$. If we take $\alpha_{3}=\frac{1}{2}, \alpha_{1}=\alpha_{2}=0$, then $\pi_{0}=\frac{1}{2}\left(\pi_{\Lambda_{3}}+\pi_{\Lambda_{5}}\right)$. Hence $\left\{\pi_{\Lambda_{1}}, \pi_{\Lambda_{4}}\right\},\left\{\pi_{\Lambda_{2}}, \pi_{\Lambda_{6}}\right\},\left\{\pi_{\Lambda_{3}}, \pi_{\Lambda_{5}}\right\}$ are probability realizers of $\underline{3}$ for $\pi_{0}$.

Remark 3. Note that, in Example $3,\left\{\Lambda_{1}, \Lambda_{2}, \Lambda_{3}\right\}$ is a realizer of an anti-chain $\underline{3}$, but $\left\{\pi_{\Lambda_{1}}, \pi_{\Lambda_{2}}, \pi_{\Lambda_{3}}\right\}$ is not a probability realizer of $\underline{3}$ for $\pi_{0}$. In fact, assume that $\pi_{0}=\alpha_{1} \pi_{\Lambda_{1}}+\alpha_{2} \pi_{\Lambda_{2}}+\alpha_{3} \pi_{\Lambda_{3}}$ for some $\alpha_{1}, \alpha_{2}, \alpha_{3} \in[0,1]$. Then we have the following equations:

$$
\begin{aligned}
& \frac{1}{2}=\pi_{0}(1,2)=\alpha_{1} 1+\alpha_{2} 1+\alpha_{3} 0, \\
& \frac{1}{2}=\pi_{0}(1,3)=\alpha_{1} 1+\alpha_{2} 0+\alpha_{3} 0, \\
& \frac{1}{2}=\pi_{0}(2,3)=\alpha_{1} 1+\alpha_{2} 0+\alpha_{3} 1, \\
& \frac{1}{2}=\pi_{0}(3,2)=\alpha_{1} 0+\alpha_{2} 1+\alpha_{3} 0 .
\end{aligned}
$$

It follows that $\alpha_{1}+\alpha_{2}=\frac{1}{2}, \alpha_{1}=\alpha_{2}=\frac{1}{2}$, which is a contradiction.

Example 4. Consider a poset $C_{2}+\underline{1}:\left.\right|_{0} ^{2} \quad \cdot 3$. This poset has three linear extensions as follows:

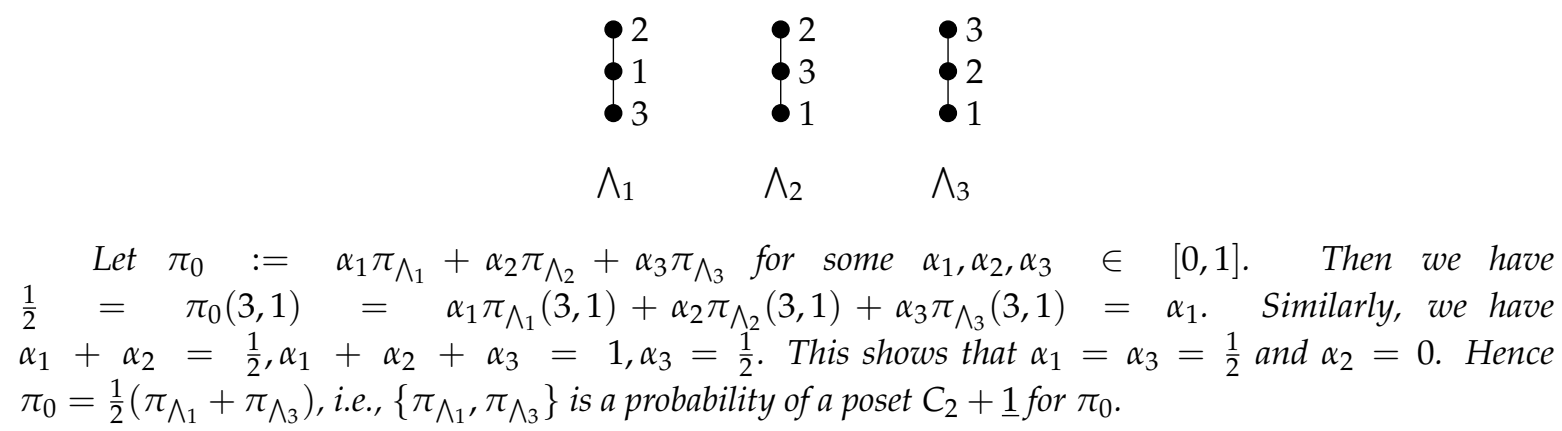

\section{Probability Functions on the Ordered Plane}

Given a plane $\mathbf{R}^{2}$, we define a partial order $\leq$ as follows: $\left(x_{1}, y_{1}\right) \leq\left(x_{2}, y_{2}\right)$ if and only if $x_{1} \leq x_{2}, y_{1} \leq y_{2}$. Such an order $\leq$ is called a product order on $\mathbf{R}^{2}$. A poset $\left(\mathbf{R}^{2}, \leq\right)$ is said to be an ordered plane (or order geometry). In the book [8], the letter $N$-poset and the letter $Y$-poset were represented as follows:
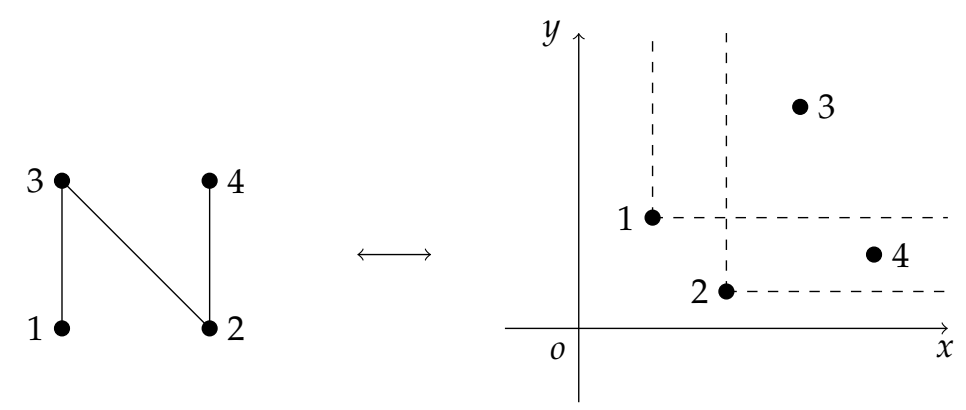

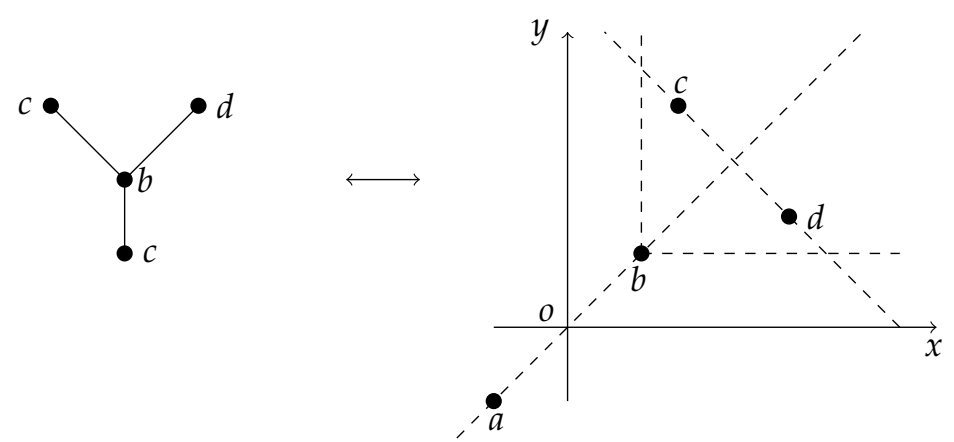

In this section, we discuss probability functions on the ordered plane. Given elements $\vec{x}, \vec{y} \in \mathbf{R}^{2}$ which are incomparable, i.e., $\vec{x} \| \vec{y}$, as below:

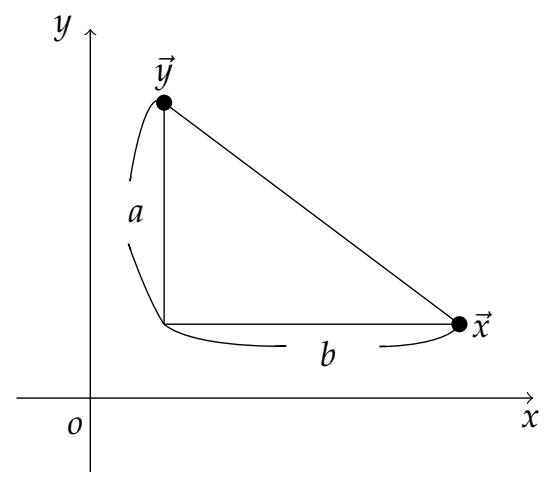

In this case, we define:

$$
\pi(\vec{x}, \vec{y}):=\frac{a}{a+b}, \pi(\vec{y}, \vec{x}):=\frac{b}{a+b} .
$$

When $\vec{x} \leq \vec{y}$, we define:

$$
\pi(\vec{x}, \vec{y}):=1, \quad \pi(\vec{y}, \vec{x}):=0
$$

Theorem 4. The mapping $\pi$ defined as (1) and (2) on $\left(\mathbf{R}^{2}, \leq\right)$ is a probability function.

Proof. The proofs of (I) and (II) are trivial, and we omit it. (III) Let $\vec{x} \neq \vec{y}$. Case (i): $\vec{x}<\vec{y}$. Then we have $\pi(\vec{x}, \vec{y})=1, \pi(\vec{y}, \vec{x})=0$. It follows that $\pi(\vec{x}, \vec{y})+\pi(\vec{y}, \vec{x})=1$. Case (ii): $\vec{x} \| \vec{y}$. We let it be drawn as:

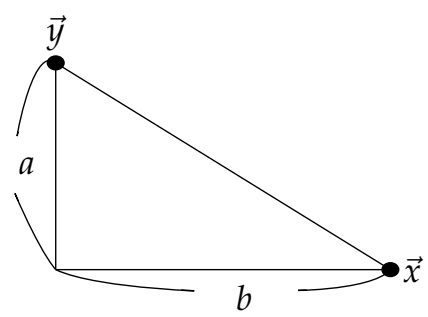


Then $\pi(\vec{x}, \vec{y})+\pi(\vec{y}, \vec{x})=\frac{a}{a+b}+\frac{b}{a+b}=1$. (IV) Let $\vec{y}<\vec{z}$ and let $\vec{x} \in \mathbf{R}^{2}$. Then we have nine subdivisions where $\vec{x}$ can be located in $\mathbf{R}^{2}$ as follows:

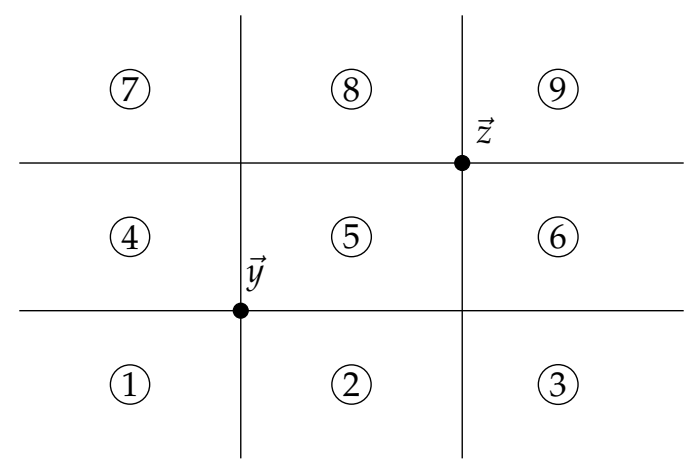

We denote its subdivision by, e.g., $\{\{1,2,3\}$, when we consider a region consisting of areas (1), (2), (3). Case (i): $\vec{x}$ lies in a subdivision $s\{1,2,4,5\}$. Then $\vec{x} \leq \vec{z}$. It follows that $\pi(\vec{x}, \vec{z})=1 \geq(\vec{x}, \vec{y})$. Case (ii): $\vec{x}$ lies in a subdivision $s\{5,6,8,9\}$. Then $\vec{y} \leq \vec{x}$ and hence $\pi(\vec{x}, \vec{y})=0 \leq(\vec{x}, \vec{z})$. Case (iii): $\vec{x}$ lies in a subdivision $s\{7\}$. Consider the following figure:

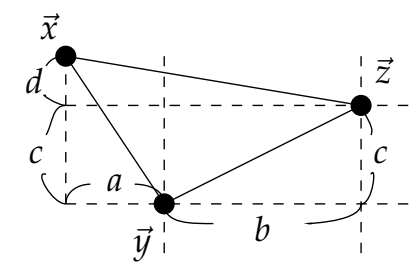

This shows that $\pi(\vec{x}, \vec{y})=\frac{a}{a+c+d}$ and $\pi(\vec{x}, \vec{z})=\frac{a+b}{a+c+d}$. Since $b>0$, we have $\pi(\vec{x}, \vec{y}) \leq(\vec{x}, \vec{z})$. Case (iv): $\vec{x}$ lies in a subdivision $s\{3\}$.

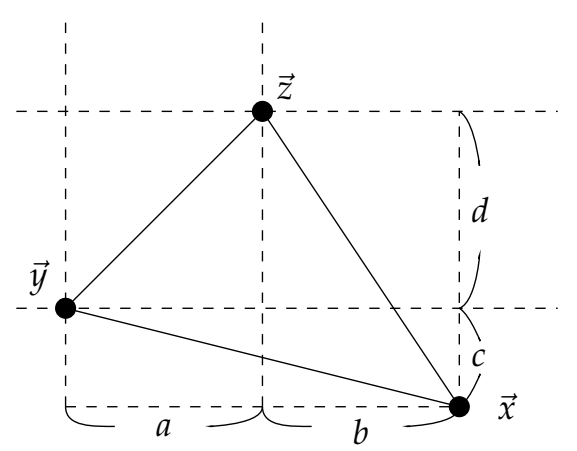

This shows that $\vec{x}\|\vec{y}, \vec{x}\| \vec{z}$, and hence $\pi(\vec{x}, \vec{y})=\frac{c}{a+b+c}, \pi(\vec{x}, \vec{z})=\frac{c+d}{b+c+d}$. The following shows that $\pi(\vec{x}, \vec{y}) \leq(\vec{x}, \vec{z})$.

$$
\begin{aligned}
\pi(\vec{x}, \vec{y}) \leq(\vec{x}, \vec{z}) & \Leftrightarrow \frac{c}{a+b+c} \leq \frac{c+d}{b+c+d} \\
& \Leftrightarrow c(b+c+d) \leq(c+d)(a+b+c) \\
& \Leftrightarrow 0 \leq a c+a d+b d: \text { true. }
\end{aligned}
$$

Hence $\pi$ is a probability function on $\left(\mathbf{R}^{2}, \leq\right)$. 
We generalize Theorem 4 by generalizing the condition (1). Let $\alpha>0, \beta>0$ with $\alpha+\beta=1$. Given elements $\vec{x}, \vec{y} \in \mathbf{R}^{2}$ which are incomparable, i.e., $\vec{x} \| \vec{y}$, as below:

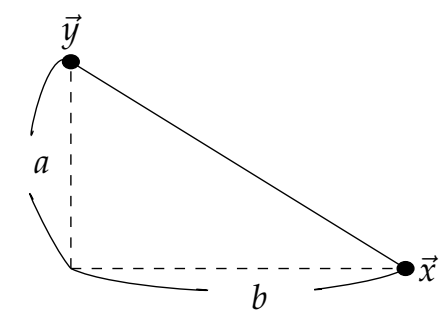

In this case, we define:

$$
\pi(\vec{x}, \vec{y}):=\frac{\alpha a}{\alpha a+\beta b}, \pi(\vec{y}, \vec{x}):=\frac{\beta b}{\alpha a+\beta b} .
$$

When $\vec{x} \leq \vec{y}$, we define $\pi(\vec{x}, \vec{y})$ and $\pi(\vec{y}, \vec{x})$ as (2) above.

Theorem 5. The mapping $\pi$ defined as (2) and (3) on $\left(\mathbf{R}^{2}, \leq\right)$ is a probability function.

Proof. The proofs of (I) (III) are easy, and we omit it. Let $\vec{y}<\vec{z}$. To show the condition (IV), we consider only two cases. Consider the following figure:



Then we have $\pi(\vec{x}, \vec{y})=\frac{\beta a}{\alpha(c+d)+\beta a}$ and $\pi(\vec{x}, \vec{z})=\frac{\beta(a+b)}{\alpha d+\beta(a+b)}$. The following shows that $\pi(\vec{x}, \vec{y}) \leq \pi(\vec{x}, \vec{z})$.

$$
\begin{aligned}
\pi(\vec{x}, \vec{y}) \leq(\vec{x}, \vec{z}) & \Leftrightarrow \frac{\beta a}{\alpha(c+d)+\beta a} \leq \frac{\beta(a+b)}{\alpha d+\beta(a+b)} \\
& \Leftrightarrow \beta a[\alpha d+\beta(a+b)] \leq \beta(a+b)[\alpha(c+d)+\beta a] \\
& \Leftrightarrow 0 \leq \alpha[(a+b) c+b d]: \text { true. }
\end{aligned}
$$

Consider the following figure:

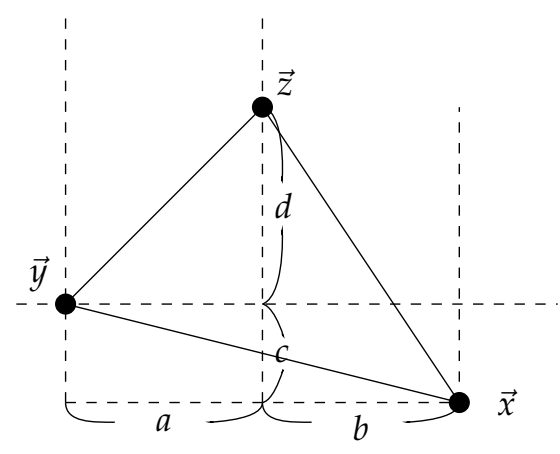


Then we have $\pi(\vec{x}, \vec{y})=\frac{\alpha c}{\alpha c+\beta(a+b)}$ and $\pi(\vec{x}, \vec{z})=\frac{\alpha(c+d)}{\alpha(c+d)+\beta b)}$. The following shows that $\pi(\vec{x}, \vec{y}) \leq \pi(\vec{x}, \vec{z})$.

$$
\begin{aligned}
\pi(\vec{x}, \vec{y}) \leq(\vec{x}, \vec{z}) & \Leftrightarrow \frac{\alpha c}{\alpha c+\beta(a+b)} \leq \frac{\alpha(c+d)}{\alpha(c+d)+\beta b} \\
& \Leftrightarrow \alpha c[\alpha(c+d)+\beta b] \leq \alpha(c+d)[\alpha c+\beta(a+b)] \\
& \Leftrightarrow \alpha \beta b c \leq \alpha \beta(c+d)(a+b) \\
& \Leftrightarrow b c \leq(c+d)(a+b): \text { true. }
\end{aligned}
$$

Hence we prove that $\pi(\vec{x}, \vec{y}) \leq \pi(\vec{x}, \vec{z})$, showing that $\pi$ is a probability function on $\left(\mathbf{R}^{2}, \leq\right)$.

Remark 4. The mapping $\pi$ discussed in Theorem 5 provides a two parameter family of probability functions on the ordered plane. If we let $\gamma:=\frac{\beta}{\alpha}$, then $\pi(\vec{x}, \vec{y})=\frac{\alpha a}{\alpha a+\beta b}=\frac{a}{a+\frac{\beta}{\alpha} b}=\frac{a}{a+\gamma b}$, i.e., we may let $\pi_{\gamma}(\vec{x}, \vec{y})=$ $\frac{a}{a+\gamma b}$, i.e., 1-parameter family of probability functions determined by $\gamma$. For example, if $\pi_{\gamma}(\vec{x}, \vec{y})=\frac{1}{2}$, then $\frac{a}{a+\gamma b}=\frac{1}{2}$. It follows that $a=\gamma b$, i.e., $-\gamma=-\frac{a}{b}$ is the slope of the lines with the property that $\pi_{\gamma}(\vec{x}, \vec{y})=\frac{1}{2}$ if and only if $\vec{x}$ and $\vec{y}$ are on such a line.

Using previous observations, we obtain more complicated probability functions such as a convex combination:

$$
\alpha_{1} \pi_{\gamma_{1}}+\alpha_{2} \pi_{\gamma_{2}}=\pi
$$

whence $\vec{x} \| \vec{y}$ yields:

$$
\pi(\vec{x}, \vec{y})=\frac{a^{2}+\left(\alpha_{1} \gamma_{2}+\alpha_{2} \gamma_{1}\right) a b}{\left(a+\gamma_{1} b\right)\left(a+\gamma_{2} b\right)}
$$

for example.

Let $\vec{x} \| \vec{y}$ in $\left(\mathbf{R}^{2}, \leq\right)$ and we say that $\vec{x}$ is below $\vec{y}$, denoted by $\vec{y} \gg \vec{x}$, if $\vec{x}$ is located below $\vec{y}$ in the ordered plane:

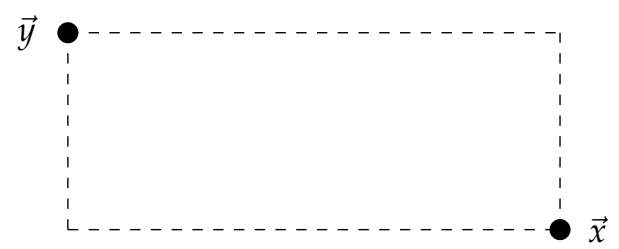

Draw a line of slope $-\left|\gamma_{i}\right|=\gamma$ through $\vec{x}$. Then this line cuts the interval $[\vec{x} \wedge \vec{y}, \vec{x} \vee \vec{y}]$ into two regions, one containing $\vec{x} \wedge \vec{y}$ and the other containing $\vec{x} \vee \vec{y}$. We have two slopes, $\gamma_{1}$ and $\gamma_{2}$ as follows:

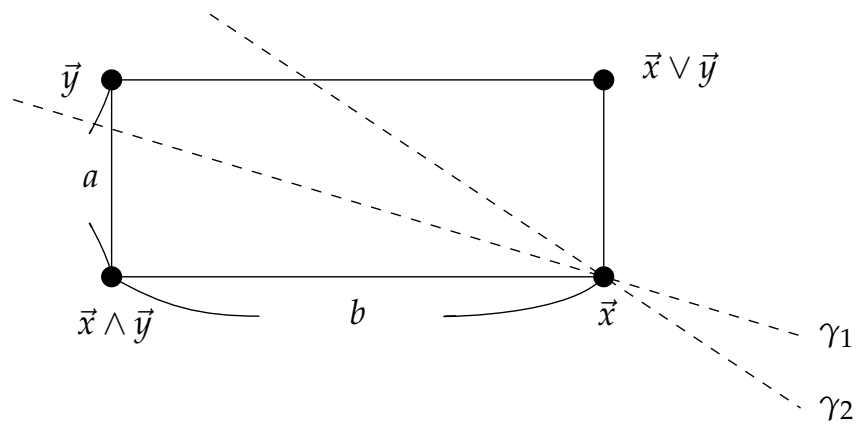


Let $A_{\gamma}$ be the region containing the point $\vec{x} \vee \vec{y}$, and let $B_{\gamma}$ be the region containing the point $\vec{x} \wedge \vec{y}$.

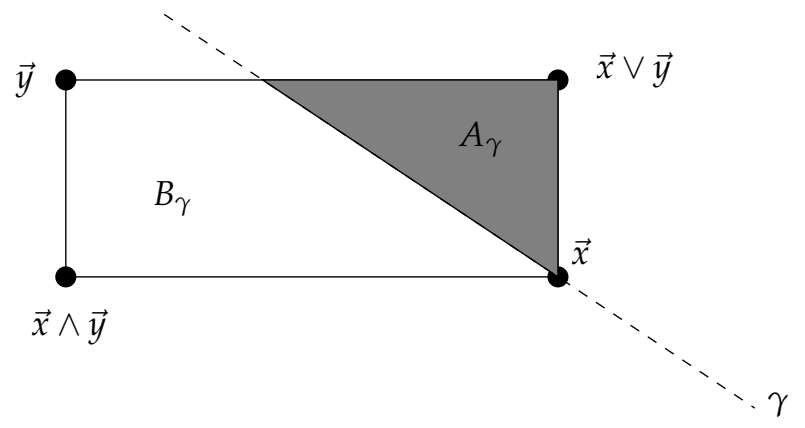

Then $A_{\gamma}+B_{\gamma}=a b$. Define $\pi_{\gamma}^{*}(\vec{x}, \vec{y}):=\frac{A_{\gamma}}{A_{\gamma}+B_{\gamma}}$ and $\pi_{\gamma}^{*}(\vec{y}, \vec{x}):=\frac{B_{\gamma}}{A_{\gamma}+B_{\gamma}}$. Then we have two cases. Case (i): $|\gamma| \leq \frac{a}{b}$.



If we take $\alpha>0$ so that $|\gamma|=\frac{\alpha}{b}$, then $B_{\gamma}=\frac{b \alpha}{2}=\frac{b^{2}}{2} \frac{\alpha}{b}=\frac{b^{2}}{2}|\gamma|$ and $A_{\gamma}=a b-\frac{b^{2}}{2}|\gamma|$. Hence $\pi_{\gamma}^{*}(\vec{x}, \vec{y})=\frac{A_{\gamma}}{a b}=1-\frac{1}{a b} \frac{b^{2}}{2}|\gamma|=1-\frac{b|\gamma|}{2 a}$ and $\pi_{\gamma}^{*}(\vec{y}, \vec{x})=\frac{B_{\gamma}}{a b}=\frac{1}{a b} \frac{b^{2}}{2}|\gamma|=\frac{|\gamma| b}{2 a}$. Notice that $\lim _{b \rightarrow 0} \pi_{\gamma}^{*}(\vec{y}, \vec{x})=\lim _{b \rightarrow 0} \frac{|\gamma| b}{2 a}=0$, which shows that $\lim _{b \rightarrow 0} \pi_{\gamma}^{*}(\vec{x}, \vec{y})=1$. Case (ii): $|\gamma| \geq \frac{a}{b}$.



If we take $\beta>0$ so that $|\gamma|=\frac{a}{\beta}$, then $A_{\gamma}=\frac{1}{2} a \beta=\frac{1}{2} a^{2} \frac{1}{|\gamma|}=\frac{a^{2}}{2|\gamma|}$ and $B_{\gamma}=a b-\frac{a^{2}}{2|\gamma|}$. Hence we have $\pi_{\gamma}^{*}(\vec{x}, \vec{y})=\frac{A_{\gamma}}{a b}=\frac{1}{a b} \frac{a^{2}}{2|\gamma|}=\frac{a}{2 b|\gamma|}$ and $\pi_{\gamma}^{*}(\vec{y}, \vec{x})=\frac{B_{\gamma}}{a b}=1-\frac{1}{2|\gamma|} \frac{a}{b}$. Also $\lim _{a \rightarrow 0} \pi_{\gamma}^{*}(\vec{x}, \vec{y})=0$ and $\lim _{a \rightarrow 0} \pi_{\gamma}^{*}(\vec{y}, \vec{x})=1$. We define a map $\pi_{\gamma}^{*}$ on the ordered plane $\left(\mathbf{R}^{2}, \leq\right)$ as follow:

$$
\pi_{\gamma}^{*}(\vec{x}, \vec{y}):= \begin{cases}1-\frac{|\gamma| b}{2 a} & \left(|\gamma| \leq \frac{a}{b}, \vec{y} \gg \vec{x}\right), \\ \frac{a}{2 b|\gamma|} & \left(|\gamma| \geq \frac{a}{b}, \vec{y} \gg \vec{x}\right) .\end{cases}
$$

and

$$
\pi_{\gamma}^{*}(\vec{y}, \vec{x}):= \begin{cases}\frac{|\gamma| b}{2 a} & \left(|\gamma| \leq \frac{a}{b}, \vec{y} \gg \vec{x}\right) \\ 1-\frac{a}{2 b|\gamma|} & \left(|\gamma| \geq \frac{a}{b}, \vec{y} \gg \vec{x}\right) .\end{cases}
$$

Moreover, we define:

$$
\pi_{\gamma}^{*}(\vec{x}, \vec{y})=1, \quad \pi_{\gamma}^{*}(\vec{y}, \vec{x})=0
$$


when $\vec{x}<\vec{y}$. We want to show that $\pi_{\gamma}^{*}$ is a probability function on the ordered plane $\left(\mathbf{R}^{2}, \leq\right)$. The proofs of the conditions (I), (II), and (III) are easy to show, and we omit it. Assume $\vec{y}<\vec{z}$. Given $\vec{x} \in \mathbf{R}^{2}$, we have nine places to put $\vec{x}$ as follows:

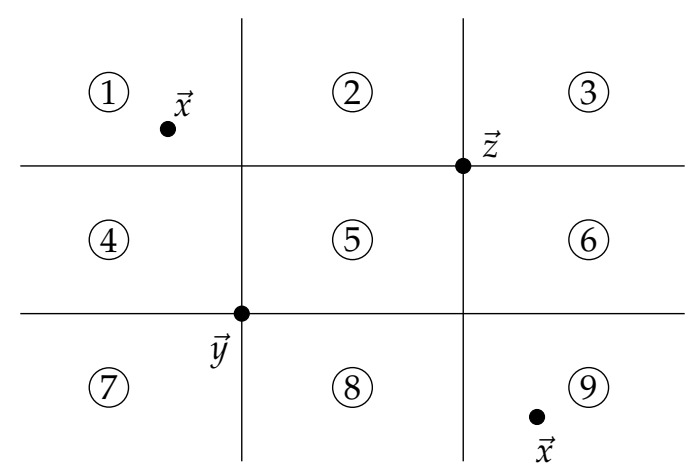

Any place except for $s\{1\}$ and $s\{9\}$ are easy to show condition (IV). Consider the case $s\{9\}$. The case can be shown as below:

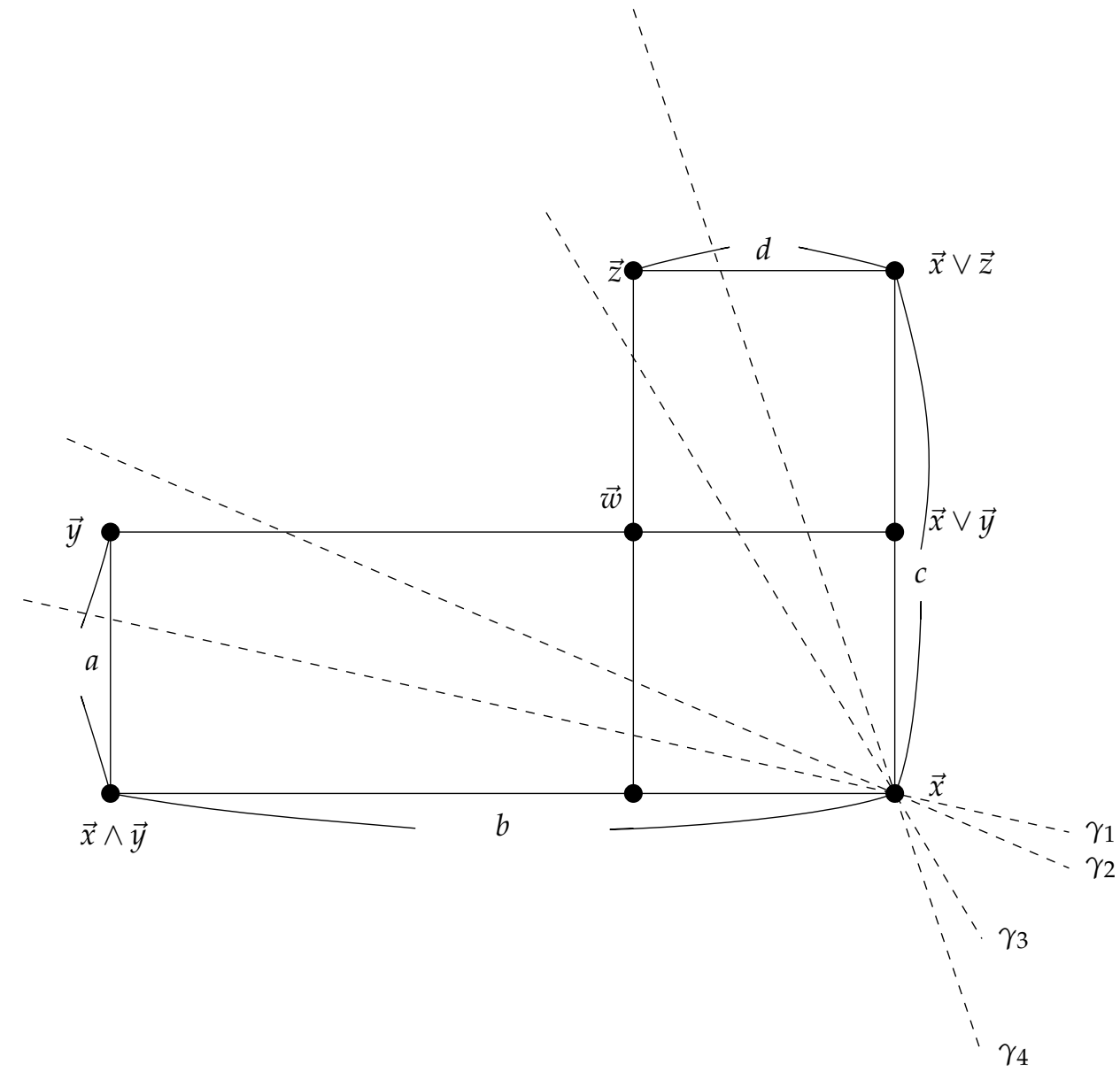

We can have four slopes between $[\vec{x} \wedge \vec{y}, \vec{x} \vee \vec{y}]$. Case (i): The slope $\gamma$ passing through the segment $[\vec{x} \wedge \vec{y}, \vec{y}]$ as follows: 


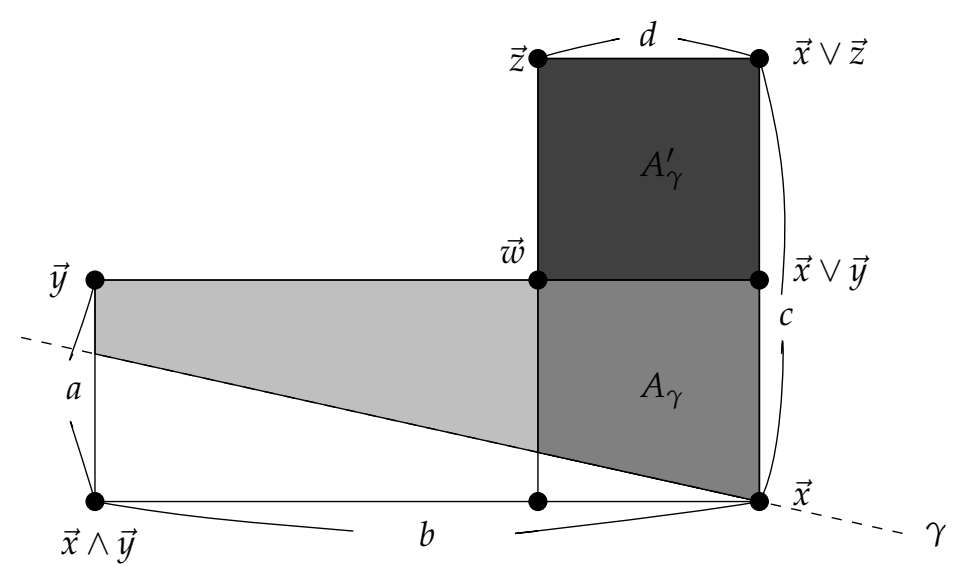

Then we have $A_{\gamma} \equiv \square$ and $A_{\gamma}^{\prime} \equiv \square$, and $B_{\gamma}=\frac{b^{2}}{2}|\gamma|$ and $B_{\gamma}^{\prime}=\frac{d^{2}}{2}|\gamma|$. Hence $\pi_{\gamma}^{*}(\vec{x}, \vec{y})=\frac{A_{\gamma}}{a b}$ and $\pi_{\gamma}^{*}(\vec{x}, \vec{z})=\frac{A_{\gamma}^{\prime}}{c d}$ proves the inequality $\pi_{\gamma}^{*}(\vec{x}, \vec{y}) \leq \pi_{\gamma}^{*}(\vec{x}, \vec{z})$ as below:

$$
\begin{aligned}
\pi_{\gamma}^{*}(\vec{x}, \vec{y}) \leq \pi_{\gamma}^{*}(\vec{x}, \vec{z}) & \Leftrightarrow \frac{A_{\gamma}}{a b} \leq \frac{A_{\gamma}^{\prime}}{c d} \\
& \Leftrightarrow \frac{B_{\gamma}}{a b} \geq \frac{B_{\gamma}^{\prime}}{c d} \\
& \Leftrightarrow \frac{1}{a b} \frac{b^{2}}{2}|\gamma| \geq \frac{1}{c d} \frac{d^{2}}{2}|\gamma| \\
& \Leftrightarrow b c \geq a d: \text { true. }
\end{aligned}
$$

Case (ii): The slope $\gamma$ passing through the segment $[\vec{y}, \vec{w}]$ as follows:

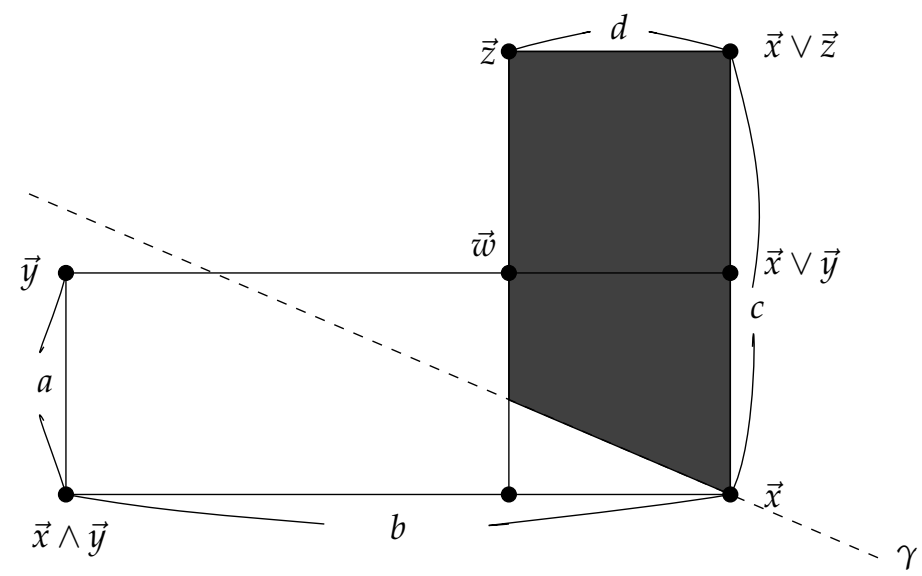


Then we have $A_{\gamma_{2}} \equiv \$ and $A_{\gamma_{2}}^{\prime} \equiv \square$ proves the inequality $\pi_{\gamma}^{*}(\vec{x}, \vec{y}) \leq \pi_{\gamma}^{*}(\vec{x}, \vec{z})$ as below:

$$
\begin{aligned}
\pi_{\gamma}^{*}(\vec{x}, \vec{y}) \leq \pi_{\gamma}^{*}(\vec{x}, \vec{z}) & \Leftrightarrow \frac{A_{\gamma}}{a b} \leq \frac{A_{\gamma}^{\prime}}{c d}=1-\frac{B_{\gamma}^{\prime}}{c d} \\
& \Leftrightarrow \frac{A_{\gamma}}{a b}+\frac{B_{\gamma}^{\prime}}{c d} \leq 1 \\
& \Leftrightarrow \frac{1}{a b} \frac{a^{2}}{2|\gamma|}+\frac{1}{c d} \frac{d^{2}|\gamma|}{2} \leq 1 \\
& \Leftrightarrow \frac{1}{2} \frac{a}{b} \frac{1}{|\gamma|}+\frac{1}{2} \frac{d}{c}|\gamma| \leq 1: \text { true, }
\end{aligned}
$$

since $\frac{a}{b} \leq|\gamma|$ and $|\gamma| \leq \frac{c}{d}$.

Case (iii): The slope $\gamma$ passing through the segment $[\vec{w}, \vec{z}]$ as follows:

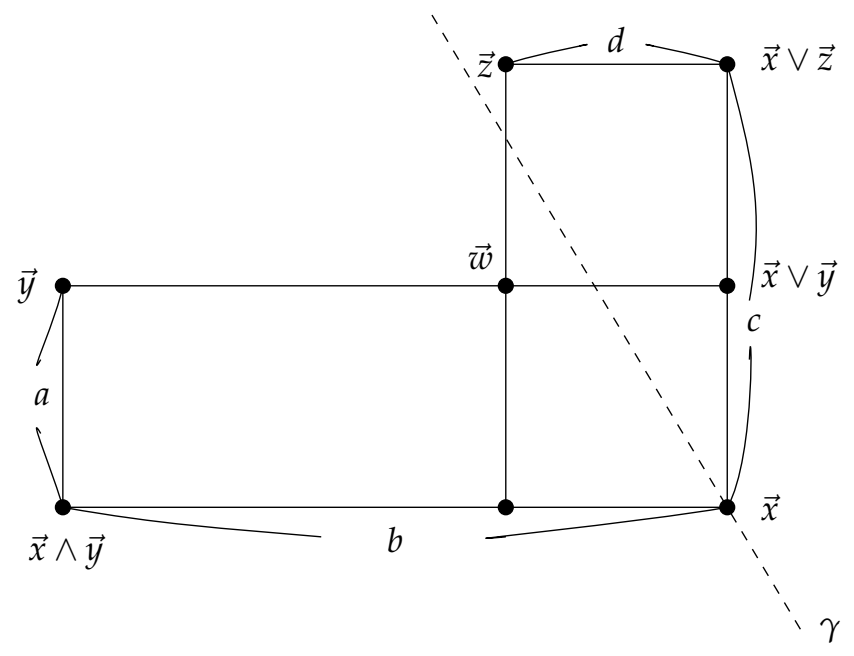

Then we have $\pi_{\gamma}^{*}(\vec{x}, \vec{y})=\frac{A_{\gamma}}{a b}=\frac{1}{a b} \frac{a^{2}}{2|\gamma|}=\frac{a}{2 b|\gamma|}$ and $\pi_{\gamma}^{*}(\vec{x}, \vec{z})=\frac{A_{\gamma}^{\prime}}{c d}=\frac{1}{c d}\left(c d-\frac{|\gamma| d^{2}}{2}\right)$. It follows that:

$$
\begin{aligned}
\pi_{\gamma}^{*}(\vec{x}, \vec{y}) \leq \pi_{\gamma}^{*}(\vec{x}, \vec{z}) & \Leftrightarrow \frac{A_{\gamma}}{a b} \leq \frac{A_{\gamma}^{\prime}}{c d}=1-\frac{1}{c d} \frac{|\gamma| d^{2}}{2} \\
& \Leftrightarrow \frac{1}{a b} \frac{a^{2}}{2|\gamma|}+\frac{1}{c d} \frac{d^{2}|\gamma|}{2} \leq 1 \\
& \Leftrightarrow \frac{1}{2} \frac{a}{b} \frac{1}{|\gamma|}+\frac{1}{2} \frac{d}{c}|\gamma| \leq 1: \text { true. }
\end{aligned}
$$

Case (iv): The slope $\gamma$ passing through the segment $[\vec{z}, \vec{x} \vee \vec{z}]$ as follows: 


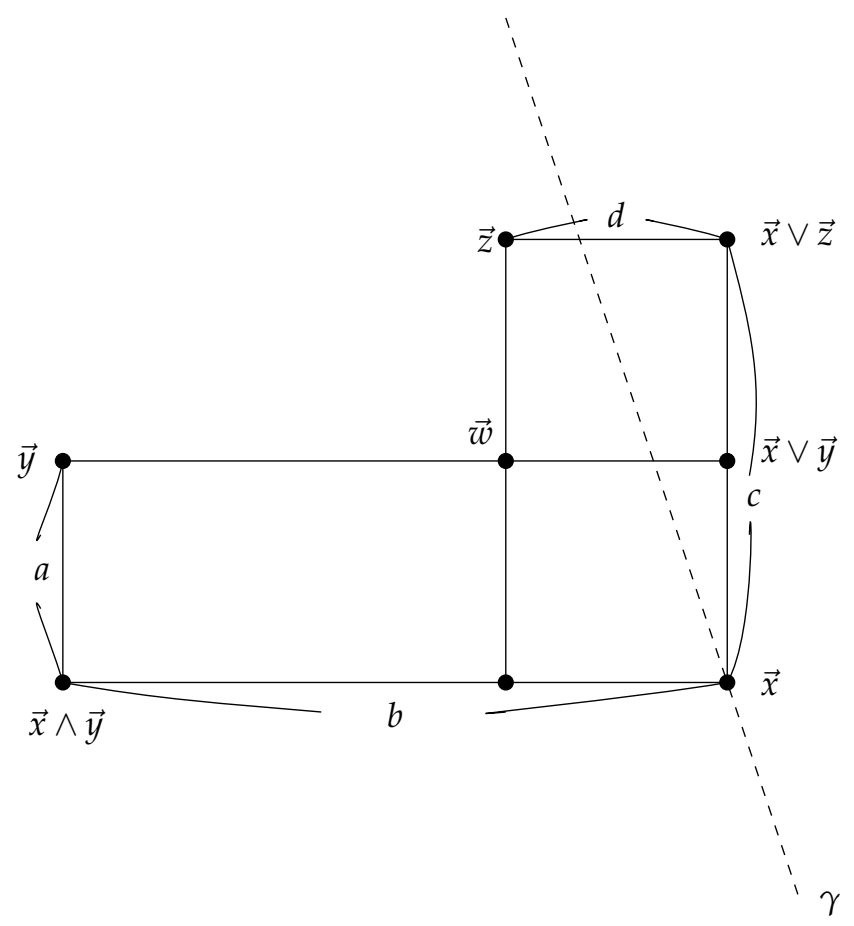

Then we have $\pi_{\gamma}^{*}(\vec{x}, \vec{y})=\frac{A_{\gamma}}{a b}=\frac{1}{a b} \frac{a^{2}}{2|\gamma|}=\frac{a}{2 b|\gamma|}$ and $\pi_{\gamma}^{*}(\vec{x}, \vec{z})=\frac{A_{\gamma}^{\prime}}{c d}=\frac{1}{c d} \frac{c^{2}}{2|\gamma|}$. It follows that:

$$
\begin{aligned}
\pi_{\gamma}^{*}(\vec{x}, \vec{y}) \leq \pi_{\gamma}^{*}(\vec{x}, \vec{z}) & \Leftrightarrow \frac{A_{\gamma}}{a b} \leq \frac{A_{\gamma}^{\prime}}{c d} \\
& \Leftrightarrow \frac{1}{a b} \frac{a^{2}}{2|\gamma|} \leq \frac{1}{c d} \frac{c^{2}}{2|\gamma|} \\
& \Leftrightarrow \frac{a}{b} \leq \frac{c}{d} \\
& \Leftrightarrow a d \leq b c: \text { true. }
\end{aligned}
$$

This shows that condition (IV) holds when $\vec{x}$ is located at the region $s\{9\}$.

Consider the region $s\{1\}$. In the following figure, there exist four slopes passing through the point $\vec{x}$.

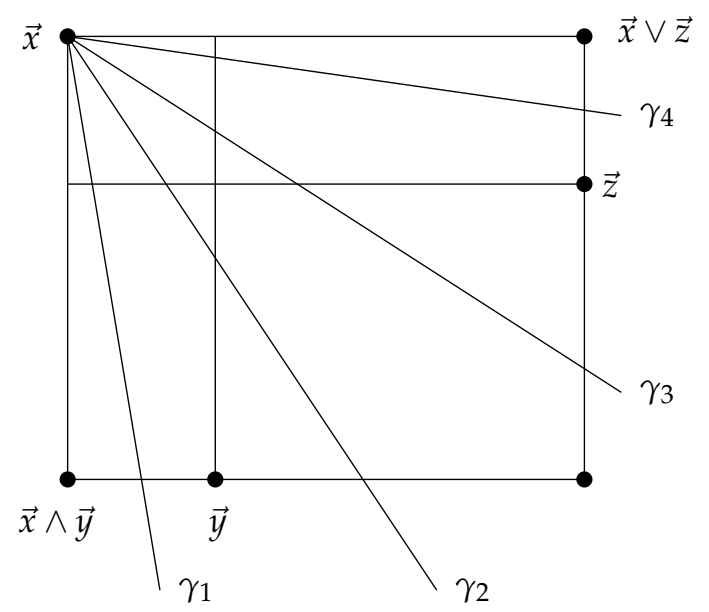


If we consider two figures below, then we find that $\pi_{\gamma_{1}}^{*}(\vec{p}, \vec{q})=\pi_{\gamma_{2}}^{*}\left(\overrightarrow{p^{\prime}}, \overrightarrow{q^{\prime}}\right)$.

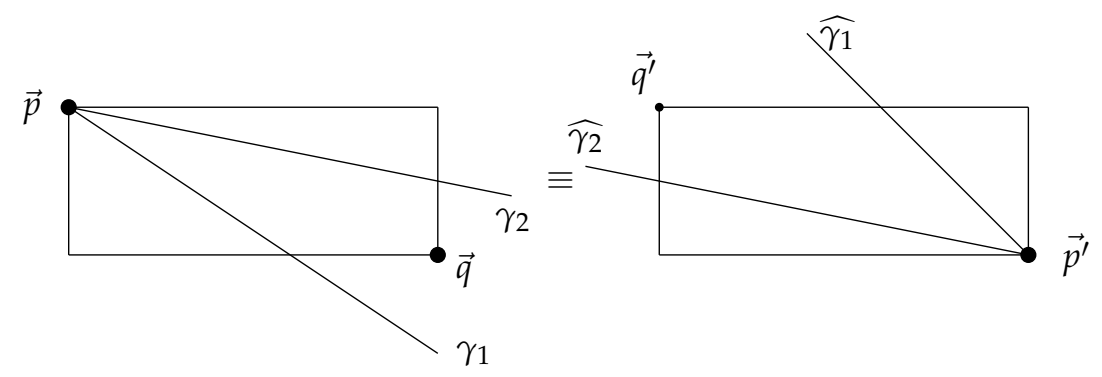

This shows that all cases for $s\{1\}$ are dual of the cases for $s\{9\}$. This shows that $\pi_{\gamma}^{*}$ is a probability function on the ordered plane $\left(\mathbf{R}^{2}, \leq\right)$. We summarize:

Theorem 6. If we define a map $\pi_{\gamma}^{*}$ on the ordered plane $\mathbf{R}^{2}$ by (4), (5), and (6), then it is a probability function on the ordered plane $\left(\mathbf{R}^{2}, \leq\right)$.

\section{Conclusions}

The notions of a trend and a probability function on $d$-algebras were developed. This kind of algebraic and axiomatic approaches gave room for the investigation on general algebraic structures in different directions. There existed algebras, e.g., $B C K$-algebras, $B C I$-algebras, which had poset structures. It is natural to consider some applications of probability functions to the poset structure. We defined the notion of a probability function on a poset, which is similar to the probability function discussed on $d$-algebras, and obtained three probability functions on posets and some basic properties. It is interesting to consider the notion of a realizer which are very useful in the study of poset theory, and so we defined a probability realizer of a poset and provided examples to describe its role for the standard probability function. We expect further investigations and theoretical results in this area. Finally, we discussed probability functions on the ordered plane and obtained probability functions acting on the ordered plane.

\section{Comments for Further Research}

(a) In Sections 4 and 5, we constructed two large families of probability functions on arbitrary finite posets, each with a particular flavor and thus also with particular possible applications. Along these lines one may extend the discussion as follows. Let a poset $(P, \leq)$ have $n$ linear extensions $\wedge_{1}, \cdots, \wedge_{n}$. Then the poset is "linearly finitary" and we construct a new poset $(Q, \leq)$ as follows:




In particular, if $P=\bigwedge_{1}$ is a chain, then $Q:=$

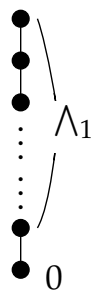

is also a chain and an ordinal sum $0 \oplus \bigwedge_{1}$. Then $Q$ may be analyzed in terms of $P$ along the line of Section 4 above.

(b) For the poset geometry, the poset $\mathbf{R}^{n}$ with $\vec{x}=\left(x_{1}, \cdots, x_{n}\right), \vec{y}=\left(y_{1}, \cdots, y_{n}\right)$ has the product order: $\vec{x} \leq \vec{y}$ if and only if $x_{i} \leq y_{i}$ for $i=1, \cdots, n$. If we analyze posets $P$ embedded in $\left(\mathbf{R}^{n}, \leq\right)$ along the lines of Section 5 , then we extend our ideas of $\left(\mathbf{R}^{2}, \leq\right)$ to that of $n$-dimensional poset geometry $\left(\mathbf{R}^{n}, \leq\right)$ and all that implies for geometry.

(c) Given a poset $(P, \leq)$ having the least element 0 , let $x * y=0$ mean $x \leq y$, and (i) $x * y=0$ mean $\pi(x, y) \geq \pi(a, b)$ for all $a, b \in P$; (ii) $\pi(x, y)<\pi\left(a_{0}, b_{0}\right)$ for some $\left(a_{0}, b_{0}\right) \in P \times P$ implies $\pi(x, y)+\pi(y, x) \geq \pi(a, b)$ for all $a, b \in P$; and (iii) $y * z=0$ implies $\pi(x, y) \leq \pi(x, z)$. Such a mapping $\pi: P \times P \rightarrow[0,1]$ (or $\pi: P \times P \rightarrow[0, \infty)$ ) is said to be a soft probability function on $(P, \leq)$ (or $(P, *))$ more generally. If we define $\pi(x, y)=\frac{2}{3}$ for all $x, y \in P$, then it is a soft probability function, but not a probability function. Thus, there are definitely differences in these concepts which may be explored further.

(d) In the setting of probability functions as studied above, the underlying model has been that of $B C K$-algebras $(X, *, 0)$ where $x * y=0$ if $x \leq y,(X, \leq)$ is a poset with minimal element 0 . Given the fact that in general the choice of 0 may be rather meaningless, we may introduce generalizations: (1) weak probability function on $(X, *): \pi: X \times X \rightarrow[0,1], \pi(x, y)+\pi(y, x)=1$ (either $x \leq y$ or $y \leq x$ analog), and (2) fuzzy weak probability function on $(X, *): \pi: X \times X \rightarrow[0,1], \pi(x, y)+\pi(y, x) \leq 1$. In either case $\pi(x, y)=1$ implies $\pi(y, x)=0$ and we may use this as an alternative to an idea, such as $x * y=0$. For a variety of modeling in applications, the groupoid $(X, *)$ may (conveniently) not be of a usual type, so that the functions $\pi$ need to be of types (1) or (2) to fit any requirements. Thus, it appears that the results obtained in this paper may be further developed for various groupoids $(X, *)$ as well as developed in (1) or (2) as described above in these comments.

Author Contributions: The authors equally contributed towards the completion of this paper.

Funding: This research received no external funding.

Acknowledgments: The authors are deeply grateful to the referees for their valuable suggestions.

Conflicts of Interest: The authors declare no conflicts of interest.

\section{References}

1. Neggers, J. Partially ordered sets and groupoids. Kyungpook Math. J. 1976, 16, 7-20.

2. Neggers, J.; Kim, H.S. Modular posets and semigroups. Semigroup Forum 1996, 53, 57-62. [CrossRef]

3. Neggers, J.; Kim, H.S. On d-algebras. Math. Slovaca 1999, 49, 19-26.

4. Cha, K.J.; Kim, H.S.; Neggers, J. Trends, probability functions and fuzzy right ideals for $d$-algebras. Comput. Math. Appl. 2011, 62, 2988-2994. [CrossRef]

5. De Loof, K.; De Baets, B.; De Meyer, H. Properties of mutual rank probabilities in partially ordered sets. In Multicirteria Ordering and Ranking: Partial Orders, Ambiguities and Applied Issues; Owsiński, J.W., Brüggemann, R., Eds.; Systems Research Institute, Polish Academy of Sciences: Warsaw, Poland, 2008; pp. 145-165. 
6. De Baets, B.; De Meyer, H.; De Loof, K. On the cycle-transitivity of the mutual rank probability relation of a poset. Fuzzy Sets Syst. 2010, 161, 2695-2708. [CrossRef]

7. Lerche, D.; Sørensen, P.B. Evaluation of the rank probabilities for partial orders based on random linear extensions. Chemosphere 2003, 53, 981-992. [CrossRef]

8. Neggers, J.; Kim, H.S. Basic Posets; World Scientific: Singapore, 1998.

(C) 2019 by the authors. Licensee MDPI, Basel, Switzerland. This article is an open access article distributed under the terms and conditions of the Creative Commons Attribution (CC BY) license (http:/ / creativecommons.org/licenses/by/4.0/). 